\title{
CHARACTERIZATIONS OF INNER PRODUCT SPACES BY INEQUALITIES INVOLVING SEMI-INNER PRODUCT
}

\author{
PAWEŁ WÓJCIK
}

Abstract. Using the notion of semi-inner product in normed spaces, in this paper we provide some new characterizations of inner product spaces. We answer a question posed by Dragomir, whether the property $(\mathrm{N})$ is characteristic for inner product spaces. We show that the space $X$ is of (N)-type if and only if the norm in $X$ comes from an inner product.

Mathematics subject classification (2010): 46C15, 46C50, 46B20, 39B62.

Keywords and phrases: Semi-inner product, characterization of inner product spaces, inequality, projection, smoothness, norm derivative.

\section{REFERENCES}

[1] C. Alsina, J. Sikorska, M. Santos Tomás, Norm Derivatives and Characterizations of Inner Product Spaces, World Scientific, Hackensack, NJ, 2009.

[2] H. F. Bohnenblust, A characterization of complex Hilbert spaces, Portugal. Math. 3 (2), (1942), 103-109.

[3] S. S. Dragomir, Semi-Inner Products and Applications, Nova Science Publishers, Inc., Hauppauge, NY, 2004.

[4] J. R. GILES, Classes of semi-inner-product spaces, Trans. Amer. Math. Soc., 129 (1967), 436-446.

[5] S. Kakutani, Some characterizations of Euclidean space, Japan. J. Math. 16 (1939), 93-97.

[6] G. LuMER, Semi-inner-product spaces, Trans. Amer. Math. Soc., 100 (1961), 29-43.

[7] P. M. Miličić, Sur la G-orthogonalité dans les espaces normés, Mat. Vesnik, 39 (1987), 325-334. 